Groups Geom. Dyn. 8 (2014), 375-389

DOI $10.4171 / \mathrm{GGD} / 230$
Groups, Geometry, and Dynamics

(C) European Mathematical Society

\title{
Finite factor representations of Higman-Thompson groups
}

\author{
Artem Dudko and Konstantin Medynets
}

\begin{abstract}
We prove that the only finite factor representations of the Higman-Thompson groups $\left\{F_{n, r}\right\}$ and $\left\{G_{n, r}\right\}$ are the regular representations and scalar representations arising from group abelianizations. As a corollary, we obtain that any measure-preserving ergodic action of the commutator subgroup of a Higman-Thompson group must be essentially free. Finite factor representations of other classes of groups are also discussed.
\end{abstract}

Mathematics Subject Classification (2010). 20E99, 47L55, 37A05.

Keywords. Higman-Thompson groups, essentially free actions, factor representations.

\section{Introduction}

One of the directions in the representation theory of infinite groups is the classification of factor representations of finite type for the group in question. In view of the Gelfand-Naimark-Segal construction, this amounts to the study of non-negativedefinite central functions (termed "characters") on the group. In [20] Vershik suggested that each indecomposable character $\chi$ (an extreme point in the simplex of characters) of a "rich" group $G$ "must" arise as $\chi(g)=\mu(\operatorname{Fix}(g)), \operatorname{Fix}(g)=\{x \in$ $X: g(x)=x\}$, for some ergodic measure-preserving action of $G$ on a measure-space $(X, \mu)$. We will refer to this suggestion as Vershik's conjecture. This conjecture was recently verified for the infinite symmetric group [21] and for the full groups of Bratteli diagrams [6].

We notice that some older results of representation theory can also be given a similar dynamical interpretation. Consider the general linear group $\mathrm{GL}_{n}\left(\mathbb{F}_{q}\right)$ over a finite field $\mathbb{F}_{q}$ and set $G_{\infty}\left(\mathbb{F}_{q}\right)=\bigcup_{n \geq 1} G_{n}\left(\mathbb{F}_{q}\right)$ with the diagonal embedding. The characters of $\mathrm{GL}_{\infty}\left(\mathbb{F}_{q}\right)$ were completely described by Skudlarek [15]. The group $\mathrm{GL}_{\infty}\left(\mathbb{F}_{q}\right)$ acts on the Cantor space $X$ of one-sided sequences over $\mathbb{F}_{q}$. The dynamical system $\left(X, \mathrm{GL}_{\infty}\left(\mathbb{F}_{q}\right)\right)$ is uniquely ergodic. Denote the invariant measure by $\mu$. Then, using the classification results of Skudlarek [15], one can check that each indecomposable character $\chi$ of $\mathrm{GL}_{\infty}\left(\mathbb{F}_{q}\right)$ has the form $\chi(g)=\mu(\operatorname{Fix}(g))^{k}$ for some $k \in\{0,1,2, \ldots\} \cup\{\infty\}$. 
The goal of the present paper is to study finite factor-representations (characters) for the Higman-Thompson family of groups $\left\{F_{n, r}\right\}$ and $\left\{G_{n, r}\right\}$ (see the definition in Section 3) and to check the validity of Vershik's conjecture for these groups. Apparently, the most famous group from this family is the Thompson group $F_{2,1}$ consisting of all piecewise linear continuous transformations of the unit interval with all singularities at points in $\left\{\frac{p}{2^{q}}: p, q \in \mathbb{N}\right\}$ and slopes in $\left\{2^{q}: q \in \mathbb{Z}\right\}$. The discussion of historical importance of these groups and their various algebraic properties can be found in [2], [3], and [16].

Our motivation is two-fold. First, these groups have been extensively studied from the group-theoretic point of view, but the theory of their representations has not been addressed yet. Our second motivation is that the actions of these groups on the spaces where they are defined have no non-atomic invariant measures, which, according to Vershik's conjecture, should imply that these groups have no non-identity/non-regular characters. The following is the main result of the present paper.

Theorem. Let $G$ be a group from the Higman-Thompson families $\left\{F_{n, r}\right\}$ or $\left\{G_{n, r}\right\}$.

(i) Let $\pi$ be a finite factor representation of $G$. Then either $\pi$ is the regular representation or $\pi$ has the form

$$
\pi(g)=\rho([g]) \mathrm{Id},
$$

where $[g]$ is the image of $g$ in the abelianization $G / G^{\prime}, G^{\prime}$ is the commutator of $G$, $\rho: G / G^{\prime} \rightarrow \mathbb{T}$ is a group homomorphism into the unit circle, and $\mathrm{Id}$ is the identity operator in some Hilbert space.

In particular, if $\chi$ is an indecomposable character of $G$, then $\chi$ is either regular or $\chi(g)=\rho([g])$, where $[g]$ is the image of $g$ in the abelianization of $G$ and $\rho: G / G^{\prime} \rightarrow$ $\mathbb{T}$ is a group homomorphism.

(ii) The commutator subgroup $G^{\prime}$ has no non-identity, non-regular characters.

This result implies that the characters of any Higman-Thompson group $G$ are convex combinations of the regular character and characters of its abelianization $G / G^{\prime}$. The structure of group characters has implications on dynamical properties of group actions. Suppose that a group $G$ admitting no non-regular/non-identity characters acts on a probability measure space $(X, \mu)$ by measure-preserving transformations. Setting $\chi(g)=\mu(\operatorname{Fix}(g))$, Fix $(g)=\{x \in X: g(x)=x\}$, one can check that the function $\chi$ is a character. This necessitates that the action must be essentially free, i.e. $\mu(\operatorname{Fix}(g))=0$ for every $g \in G \backslash\{e\}$, see Theorem 2.12 and Corollary 3.10 for details.

Corollary. Let $G$ be the commutator subgroup of a group from the Higman-Thompson families $\left\{F_{n, r}\right\}$ or $\left\{G_{n, r}\right\}$. Then any faithful measure-preserving ergodic action of $G$ on a probability measure space $(X, \mu)$ is essentially free.

Combining this result with [1], Proposition 14, we obtain the following. Note that the commutator subgroups of groups in $\left\{F_{n, r}\right\}$ or $\left\{G_{n, r}\right\}$ are simple [2], [8]. 
Corollary. Let $G$ be the commutator subgroup of a group from the Higman-Thompson families $\left\{F_{n, r}\right\}$ or $\left\{G_{n, r}\right\}$. Then $G$ has no non-trivial invariant random subgroup.

In our proofs, we mostly utilize the fact that the commutators of Higman-Thompson groups have no non-atomic invariant measures on the spaces over which they are defined. This means that the orbit equivalence relations generated by their actions are compressible [9]. This observation allows us to state the main result in terms of dynamical properties of group actions (Theorems 2.9 and 2.11) and extend it to other classes of transformation groups (Section 3).

We notice that the amenability of the Thompson group $F_{2,1}$ is still an open question. Thus, it would be interesting to relate the constraints on group actions coming from the absence of non-trivial characters to the amenability. We would like to mention though that there are amenable groups with no non-identity/non-regular characters. In [13], [14] Ovchinnikov showed that every Chevalier group $G=L(K)$ over an infinite discrete field $K$ admits only the trivial and the regular indecomposable characters. We notice that for some discrete fields the associated Chevalier groups are locally finite.

The structure of the paper is the following. In Section 2 we develop the general theory of finite type factor representations for groups admitting compressible actions. In Section 3 we apply our general results to the Higman-Thompson groups and to the full groups of irreducible shifts of finite type [11].

To complete the introduction, we mention that finite type representations have been completely studied only for a handful of infinite groups. Amongst these, the groups mentioned above, the infinite unitary group [18] and [19], and the general linear group $\mathrm{GL}(n, K)$ over an infinite discrete field [10].

\section{General theory}

In this section we show that if a group admits a compressible action on a topological space, then this group, under some algebraic assumptions, has no non-trivial factor representations. We will start with definitions from the representation theory of infinite groups.

Definition 2.1. A character of a group $G$ is a function $\chi: G \rightarrow \mathbb{C}$ satisfying the following conditions:

1) $\chi\left(g_{1} g_{2}\right)=\chi\left(g_{2} g_{1}\right)$ for any $g_{1}, g_{2} \in G$;

2) the matrix $\left\{\chi\left(g_{i} g_{j}^{-1}\right)\right\}_{i, j=1}^{n}$ is non-negatively defined for any $n$ and $g_{1}, \ldots$, $g_{n} \in G$

3) $\chi(e)=1$. Here $e$ is the group identity.

A character $\chi$ is called indecomposable if it cannot be written in the form $\chi=$ $\alpha \chi_{1}+(1-\alpha) \chi_{2}$, where $0<\alpha<1$ and $\chi_{1}, \chi_{2}$ are distinct characters. 
For a unitary representation $\pi$ of a group $G$ denote by $\mathcal{M}_{\pi}$ the $W^{*}$-algebra generated by the operators of the representation $\pi$. Recall that the commutant $S^{\prime}$ of a set $S$ of operators in a Hilbert space $H$ is the algebra $S^{\prime}=\{A \in B(H): A B=$ $B A$ for any $B \in S\}$.

Definition 2.2. A representation $\pi$ of a group $G$ is called a factor representation if the algebra $\mathcal{M}_{\pi}$ is a factor, that is $\mathcal{M}_{\pi} \cap \mathcal{M}_{\pi}^{\prime}=\mathbb{C}$ Id.

The indecomposable characters on a group $G$ are in one-to-one correspondence with the finite type factor representations of $G$. The classification of factors into types can be found in Chapter 5 of [17]. Starting with an indecomposable character $\chi$ on $G$ one can construct a triple $(\pi, H, \xi)$, referred to as the Gelfand-Naimark-Segal (GNS for short) construction. Here $\pi$ is a finite type factor representation acting in the space $H$, and $\xi$ is a unit vector in $H$ such that $\chi(g)=(\pi(g) \xi$, $\xi)$ for every $g \in G$ (see, for example, [6], Section 2.3). Note that the vector $\xi$ is cyclic and separating for the von Neumann algebra $\mathcal{M}_{\pi}$. The latter means that if $A \xi=0$, for some $A \in \mathcal{M}_{\pi}$, then $A=0$.

Remark 2.3. We note that each character defines a factor representation up to quasi-equivalence. Two unitary representations $\pi_{1}$ and $\pi_{2}$ of the same group $G$ are called quasi-equivalent if there is an isomorphism of von Neumann algebras $\omega: \mathcal{M}_{\pi_{1}} \rightarrow \mathcal{M}_{\pi_{2}}$ such that $\omega\left(\pi_{1}(g)\right)=\pi_{2}(g)$, for each $g \in G$. For example, all $\mathrm{II}_{1}$ factor representations of an amenable group are hyperfinite [4] (Corollary 6.9 and Theorem 6) and, hence, generate isomorphic algebras. At the same time, they might be not quasi-equivalent.

Suppose that $G$ is an infinite conjugacy class (abbr. ICC) group. Then its left regular representation $\pi$ generates a $\mathrm{II}_{1}$-factor and the function $\chi(g)=\left(\pi(g) \delta_{e}, \delta_{e}\right)$ is an indecomposable character (termed the regular character).

Definition 2.4. We say that a group $H$ has no proper characters if $\chi$ being an indecomposable character of $H$ implies that either $\chi$ is the identity character given by

$$
\chi(g)=1 \quad \text { for every } g \in G
$$

or the regular character defined as

$$
\chi(g)=0 \quad \text { if } g \neq e \text { and } \chi(e)=1 .
$$

We notice that for non-ICC groups the regular characters are decomposable.

Fix a regular Hausdorff topological space $X$. Notice that any two distinct points of $X$ have open neighborhoods with disjoint closures. To exclude trivial counterexamples to our statements we assume that the set $X$ is infinite. Suppose that a group $G$ acts on $X$. For a group element $g \in G$, denote its support by $\operatorname{supp}(g)=\overline{\{x \in X: g(x) \neq x\}}$. 
Definition 2.5. We say that the action of $G$ on $X$ is compressible if there exists a basis $\mathcal{U}$ of the topology on $X$ such that

(i) for all $g \in G$, there exists $U \in \mathfrak{U}$ such that $\operatorname{supp}(g) \subset U$;

(ii) for all $U_{1}, U_{2} \in \mathfrak{U}$, there exists $g \in G$ such that $g\left(U_{1}\right) \subset U_{2}$;

(iii) for all $U_{1}, U_{2}, U_{3} \in \mathfrak{U}$ with $\bar{U}_{1} \cap \bar{U}_{2}=\varnothing$, there exists $g \in G$ such that $g\left(U_{1}\right) \cap U_{3}=\varnothing$ and $\operatorname{supp}(g) \cap U_{2}=\varnothing$.

(iv) for all $U_{1}, U_{2} \in \mathfrak{U}$, there exists $U_{3} \in \mathfrak{U}$ such that $U_{3} \supset U_{1} \cup U_{2}$.

Remark 2.6. Suppose that $X$ is a Polish space. If an action of $G$ on $X$ is compressible, then the $G$-action has no probability invariant measure. The latter is equivalent to the $G$-orbit equivalence relation being compressible (see [9] and references therein). This observation motivates our terminology.

The following result relates the dynamical properties of group actions to the functional properties of group characters.

Proposition 2.7. Let $G$ be a countable group admitting a compressible action by homeomorphisms on some regular Hausdorff topological space $X$. Then for every non-regular indecomposable character $\chi$ of $G$ there exists $g \neq e$ such that $|\chi(g)|=1$.

Proof. Consider a proper indecomposable character $\chi$ of $G$. Assume that $|\chi(g)|<1$ for all $g \neq e$. Let $(\pi, H, \xi)$ be the GNS-construction associated to $\chi$.

(1) We notice that the definition of the compressible action implies that $\chi$ has the multiplicativity property in the sense that if $U_{1}, U_{2} \in \mathfrak{U}$ and $g, h \in G$ are such that

$$
\operatorname{supp}(g) \subset U_{1}, \quad \operatorname{supp}(h) \subset U_{2} \quad \text { and } \quad \bar{U}_{1} \cap \bar{U}_{2}=\varnothing,
$$

then

$$
\chi(g h)=\chi(g) \chi(h) .
$$

Indeed, find an increasing sequence of finite sets $F_{n} \subset G$ with $\bigcup_{n} F_{n}=G$. Then by condition (i) and condition (iv) of Definition 2.5, we can find open sets $V_{n} \in \mathfrak{U}$ such that

$$
V_{n} \supset \bigcup_{f \in F_{n}} \operatorname{supp}(f) .
$$

By condition (iii) there exist elements $r_{n} \in G$ such that

$$
r_{n}\left(U_{1}\right) \cap V_{n}=\varnothing \quad \text { and } \operatorname{supp}\left(r_{n}\right) \cap U_{2}=\varnothing .
$$

Then $r_{n} h r_{n}^{-1}=h$ and $\operatorname{supp}\left(r_{n} g r_{n}^{-1}\right) \cap \operatorname{supp}(f)=\varnothing$ for every $f \in F_{n}$. Passing to a subsequence if needed, we can assume that $\pi\left(r_{n} g r_{n}^{-1}\right)$ converges weakly to an operator $Q \in \mathcal{M}_{\pi}$. Notice that the trace $\tau(Q)$ is equal to $\chi(g)$. Since the operator 
$Q$ commutes with $\pi\left(F_{n}\right)$ for every $n$, we obtain that $Q$ belongs to the center of $\mathcal{M}_{\pi}$. Therefore, $Q$ is scalar and $Q=\chi(g)$ Id. Thus

$$
\chi(g h)=\lim _{n \rightarrow \infty}\left(\pi\left(r_{n} g h r_{n}^{-1}\right) \xi, \xi\right)=(Q \pi(h) \xi, \xi)=\chi(g) \chi(h) .
$$

(2) We claim that, for every $\varepsilon>0$ and every open set $U$ there exists $g \in G$ with $\operatorname{supp}(g) \subset U$ and $|\chi(g)|<\varepsilon$. Indeed, fix an element $h \neq e$ and $n \in \mathbb{N}$. Find $n$ subsets $V_{1}, \ldots, V_{n} \in \mathfrak{U}$ such that $\bar{V}_{j} \cap \bar{V}_{k}=\varnothing$, for $j \neq k$. By condition (i) and condition (ii) we can choose elements $g_{1}, \ldots, g_{n} \in G$ such that $g_{j}(\operatorname{supp}(h)) \subset V_{j}$, for each $j$. Set

$$
f=\left(g_{1} h g_{1}^{-1}\right)\left(g_{2} h g_{2}^{-1}\right) \cdots\left(g_{n} h g_{n}^{-1}\right) .
$$

By multiplicativity, we obtain that $\chi(f)=\chi(h)^{n}$. Choosing $n$ sufficiently large we obtain an element $f$ with $|\chi(f)|<\varepsilon$. By condition (i) and condition (ii) we can find an element $g$ conjugate to $f$ with $\operatorname{supp}(g) \subset U$, which proves the claim.

(3) Consider an element $g \in G, g \neq e$. Find an open set $U$ with $\overline{g(U)} \cap \bar{U}=\varnothing$. Fix $\varepsilon>0$ and $n \in \mathbb{N}$. Using condition (ii) and (iv) of Definition 2.5, we can find subsets $U_{1}, \ldots, U_{n}, V_{1}, \ldots, V_{n} \in \mathfrak{U}$ with pairwise disjoint closures such that $g\left(V_{i}\right) \subset U_{i} \subset U$ for each $i$. Find $h_{j} \in G, j=1, \ldots, n$, supported by $U_{j}$ with $\left|\chi\left(h_{j}\right)\right|<\varepsilon$. Set $\xi_{j}=\pi\left(h_{j} g h_{j}^{-1}\right) \xi$. Then for $i \neq j$, the multiplicativity of $\chi$ implies that

$$
\begin{aligned}
\left(\xi_{i}, \xi_{j}\right) & =\chi\left(h_{j} g^{-1} h_{j}^{-1} h_{i} g h_{i}^{-1}\right) \\
& =\chi\left(h_{j}\left(g^{-1} h_{j}^{-1} g\right)\left(g^{-1} h_{i} g\right) h_{i}^{-1}\right) \\
& =\chi\left(h_{j}\right) \chi\left(g^{-1} h_{j}^{-1} g\right) \chi\left(g^{-1} h_{i} g\right) \chi\left(h_{i}^{-1}\right) .
\end{aligned}
$$

As $\left|\chi\left(h_{j}\right)\right|<\varepsilon$, we obtain that $\left|\left(\xi_{j}, \xi_{i}\right)\right|<\varepsilon$. Thus,

$$
\left\|\xi_{1}+\ldots+\xi_{n}\right\| \leq(n+n(n-1) \varepsilon)^{\frac{1}{2}} .
$$

Since $\left(\xi_{l}, \xi\right)=\chi(g)$ for each $l$, we have

$$
|\chi(g)|=\frac{1}{n}\left|\left(\xi_{1}+\xi_{2}+\ldots+\xi_{n}, \xi\right)\right| \leq \frac{1}{n}(n+n(n-1) \varepsilon)^{\frac{1}{2}} .
$$

When $n$ goes to infinity, we obtain

$$
|\chi(g)| \leq \varepsilon^{\frac{1}{2}} .
$$

Since $\varepsilon>0$ is arbitrary, we conclude that $\chi(g)=0$. Thus, $\chi$ is the regular character.

Lemma 2.8. Let $G$ be a simple group and $\chi$ be a character. If $|\chi(g)|=1$ for some $g \in G, g \neq e$, then

$$
\chi(s)=1 \text { for all } s \in G .
$$

Equivalently, if $\chi$ is not the identity character, then $|\chi(s)|<1$ for all $s \neq e$. 
Proof. Let $c=\chi(g),|c|=1$. Consider the GNS construction $(\pi, H, \xi)$ corresponding to $\chi$. Using the Cauchy-Schwarz inequality and the fact that the vector $\xi$ is separating, we obtain that

$$
(\pi(g) \xi, \xi)=c \Longrightarrow \pi(g) \xi=c \xi \Longrightarrow \pi(g)=c \mathrm{Id} .
$$

Take an arbitrary element $h \in G$ which does not commute with $g$ and set $s=$ $h g h^{-1} g^{-1}$. Then $\pi(s)=$ Id. It follows that $\pi\left(s_{1}\right)=$ Id for all $s_{1}$ from the normal subgroup generated by $s$. Since $G$ is simple, we obtain that $\pi(g)=$ Id for every $g \in G$. Thus, $\chi$ is the identity character.

As a corollary of Lemma 2.8 and Proposition 2.7, we immediately obtain the following result.

Theorem 2.9. Let $G$ be a simple countable group admitting a compressible action on a regular Hausdorff topological space $X$. Then $G$ has no proper characters.

Remark 2.10. Jesse Peterson [private communications] noticed that the proof of Proposition 2.7, in fact, implies that if $\chi$ is not a regular character, then $|\chi(g)|=1$ for all $g \in G \backslash\{e\}$. Since $1=|\chi(g)|=|(\pi(g) \xi, \xi)| \leq 1$, by the Cauchy-Schwarz inequality, we obtain that $\pi(g) \xi=c_{g} \xi$ for some $\left|c_{g}\right|=1$. This yields that $\chi$ is a homomorphism of $G$ into the unit circle. Thus, if a group (not necessarily simple) $G$ admitting a compressible action has no homomorphisms into the unit circle, then $G$ has no proper characters. We notice that perfect (and, in particular, simple) groups have no non-trivial homomorphisms into the unit circle.

Let $G$ be a group. For a subgroup $R$ of $G$ and an element $g \in G$ set $C_{R}(g)=$ $\left\{h g h^{-1}: h \in R\right\}$. Denote by $N(R)$ the normal closure of $R$ in $G$, i.e., the subgroup of $G$ generated by all elements of the form $\mathrm{grg}^{-1}, g \in G, r \in R$.

Theorem 2.11. Let $G$ be a group and $R$ be an ICC subgroup of $G$ such that

(i) $R$ has no proper characters;

(ii) for every $g \in G \backslash\{e\}$, there exists a sequence of distinct elements $\left\{g_{i}\right\}_{i \geq 1} \subset$ $C_{R}(g)$ such that $g_{i}^{-1} g_{j} \in R$ for any $i, j$.

Then each finite type factor representation $\pi$ of $G$ is either regular or has the form

$$
\pi(g)=\omega([g]),
$$

where $\omega$ is a finite type factor representation of $G / N(R)$ and $[g] \in G / N(R)$ is the coset of the element $g$.

Proof. Consider an indecomposable character $\chi$ of $G$. Let $(\pi, H, \xi)$ be the GNSconstruction associated to $\chi$. 
(1) Consider the restriction of $\pi$ onto the subgroup $R$. Set $H_{R}=\overline{\operatorname{Lin}(\pi(R) \xi)}$. Since the restriction of $\chi$ on $R$ is a character and the only indecomposable characters of the group $R$ are the regular and the identity characters, we can decompose the space $H_{R}$ into $R$-invariant subspaces $H_{1}$ and $H_{2}$ (possibly trivial) such that $H_{R}=$ $H_{1} \bigoplus H_{2}$ with $\pi(R) \mid H_{1}$ being the identity representation and $\pi(R) \mid H_{2}$ being the regular representation.

The orthogonal projections $\left\{P_{i}\right\}$ onto $H_{i}, i=1,2$ belong to the center of the algebra generated by $\pi(R)$. In particular, $P_{i}$ lies in the algebra $\mathcal{M}_{\pi}$. Furthermore,

$$
\chi(g)=\alpha \chi_{\mathrm{id}}(g)+(1-\alpha) \chi_{\mathrm{reg}}(g) \text { for all } g \in R,
$$

where $\chi_{\text {id }}$ is the identity character, $\chi_{\text {reg }}$ is the regular character, and $\alpha \in[0,1]$. If $\alpha \neq 0,1$, we can write down the vector $\xi$ as

$$
\xi=\alpha^{\frac{1}{2}} \xi_{1}+(1-\alpha)^{\frac{1}{2}} \xi_{2},
$$

where $\xi_{1} \in H_{1}, \xi_{2} \in H_{2}$ are unit vectors such that

$$
\left(\pi(h) \xi_{1}, \xi_{1}\right)=\chi_{\mathrm{id}}(h)=1, \quad\left(\pi(h) \xi_{2}, \xi_{2}\right)=\chi_{\mathrm{reg}}(h)=\delta_{h, e} \quad \text { for all } h \in R
$$

For convenience, if $\alpha=0$, we set $\xi_{1}=0, \xi_{2}=\xi$, and if $\alpha=1$, we set $\xi_{1}=\xi$, $\xi_{2}=0$. Observe that $H_{i}=\overline{\operatorname{Lin}\left(\pi(R) \xi_{i}\right)}, i=1,2$.

(2) Assume that $H_{2} \neq\{0\}$. Consider an arbitrary element $g \in G, g \neq e$. By our assumptions there exists a sequence of elements $\left\{h_{n}\right\} \in R \backslash\{e\}$ such that $h_{m}^{-1} g^{-1} h_{m} h_{n}^{-1} g h_{n} \in R$, for all $n$ and $m$, and the elements $h_{n}^{-1} g h_{n}$ are pairwise distinct. Set $g_{m}=h_{m}^{-1} g h_{m}$. Since $g_{m}^{-1} g_{n} \in R \backslash\{e\}$, we obtain that

$$
\left(\pi\left(g_{n}\right) \xi_{2}, \pi\left(g_{m}\right) \xi_{2}\right)=\chi_{\mathrm{reg}}\left(g_{m}^{-1} g_{n}\right)=0 .
$$

This shows that $\pi\left(g_{m}\right) \xi_{2} \rightarrow 0$ weakly. Observe also that

$$
\begin{aligned}
\left(\pi\left(g_{n}\right) \xi_{2}, \xi_{2}\right) & =\left(\pi\left(g_{n}\right) P_{2} \xi, P_{2} \xi\right)=\tau\left(P_{2} \pi\left(h_{n}^{-1} g h_{n}\right) P_{2}\right) \\
& =\tau\left(\pi\left(h_{n}^{-1}\right) P_{2} \pi(g) P_{2} \pi\left(h_{n}\right)\right)=\tau\left(P_{2} \pi(g) P_{2}\right) .
\end{aligned}
$$

Since the latter is independent of $n$ and $\pi\left(g_{n}\right) \xi_{2} \rightarrow 0$, we conclude that

$$
\left(\pi(g) \xi_{2}, \xi_{2}\right)=\tau\left(P_{2} \pi(g) P_{2}\right)=0 .
$$

Set $H_{0}=\overline{\operatorname{Lin}\left(\pi(G) \xi_{2}\right)}$. Then $\pi(G) \mid H_{0}$ is quasi-equivalent to the regular representation. Since $\pi$ is a factor representation, we conclude that $\pi$ is the regular representation.

(3) Assume that $H_{2}=\{0\}$. Then $\xi=\xi_{1}$ and $\pi(h)=$ Id for every $h \in R$. Therefore, $\pi(g)=$ Id for all $g \in N(R)$. This means that the representation $\pi$ factors through the quotient $G / N(R)$ and defines a finite type factor representation $\omega$ of $G / N(R)$ such that $\pi(g)=\omega([g])$ for all $g \in G$. 
Recall that a finite type factor representation of a group $G$ is of type $\mathrm{I}$ if the von Neumann algebra of the representation is isomorphic to the algebra of all linear operators in some finite-dimensional Hilbert space. We say that an action of group $G$ on a measure space $(Y, \mu)$ is trivial if $g(x)=x$ for every $g \in G$ and $\mu$-almost every $x \in X$. The following result shows that any ergodic action of a group admitting no non-identity/non-regular characters must be essentially free, that is $\mu(\operatorname{Fix}(g))=0$ for all $g \in G \backslash\{e\}$.

Theorem 2.12. Assume that every finite type factor representation of a countable ICC group $G$ is either regular or of type I and that there is at most countably many (up to quasi-equivalence) finite type factor representations of $G$. Then every faithful ergodic measure-preserving action of $G$ is essentially free.

Proof. Consider an ergodic action of $G$ on a measure space $(Y, \mu)$. Set

$$
\tilde{Y}=\{(x, y) \in Y \times Y \mid x=g(y) \text { for some } g \in G\} .
$$

For a Borel set $A \subset \tilde{Y}$ and a point $x \in Y$, set $A_{x}=\{(x, y) \in A\}$. Define a $\sigma$-finite measure $\tilde{\mu}$ on $\tilde{Y}$ by $\tilde{\mu}(A)=\int_{Y} \operatorname{card}\left(A_{x}\right) d \mu(x)$. Given a function $f \in L^{2}(\tilde{Y}, \tilde{\mu})$ and a group element $g \in G$, set

$$
(\pi(g) f)(x, y)=f\left(g^{-1} x, y\right) .
$$

Then $\pi(g)$ is a unitary operator on the Hilbert space $L^{2}(\tilde{Y}, \tilde{\mu})$. Denote by $\xi$ the indicator function of the diagonal of $Y \times Y$. Set $H=\overline{\operatorname{Lin}\{\pi(G) \xi\}}$. We note the von Neumann algebra $\mathcal{M}_{\pi}$ generated by $\pi(G)$, restricted to $H$, is of finite type. We refer the reader to [5] for details. Since the group $G$ has at most countably many finite factor representations, the representation $\pi$ decomposes into a direct sum (at most countable) of factor representations.

Our goal is to show that the representation $\pi$ is regular. Then the uniqueness of the trace implies that $(\pi(g) \xi, \xi)=0$ for every $g \neq e$. Using the identity $\mu(\operatorname{Fix}(g))=$ $(\pi(g) \xi, \xi)$, we obtain that the action is essentially free.

Suppose to the contrary that the decomposition of $\pi$ into factors contains a non-regular factor representation $\pi_{1}$, which, by our assumptions, generates a finitedimensional von Neumann factor. Let $P_{1}$ be a projection from the center of $\mathcal{M}_{\pi}$ such that $\pi_{1}(g)=P_{1} \pi(g)$, for every $g \in G$. Set $\xi_{1}=P_{1} \xi$.

Since for every $g \in G$ the unitary operator $\left(\pi^{\prime}(g) f\right)(x, y)=f\left(x, g^{-1} y\right)$ belongs to $\mathcal{M}_{\pi}^{\prime}$ and $\pi^{\prime}(g) \xi=\pi\left(g^{-1}\right) \xi$, we have that

$$
\pi^{\prime}(g) \pi(g) \xi_{1}=\pi^{\prime}(g) \pi(g) P_{1} \xi=P_{1} \pi^{\prime}(g) \pi(g) \xi=P_{1} \xi=\xi_{1},
$$

for all $g \in G$. This implies that the function $h(x):=\left|\xi_{1}(x, x)\right|$ is $G$-invariant and $\mu$-integrable on $Y$. By ergodicity, we obtain that $h(x) \equiv C$ on $Y$, for some constant $C$. Note that if $C=0$, then

$$
0=\int_{\widetilde{Y}} \xi_{1}(x, y) \xi(x, y) d \tilde{\mu}(x, y)=\left(\xi_{1}, \xi\right),
$$


which is impossible as the projection of $\xi$ onto $\xi_{1}$ is non-trivial.

Fix an orthonormal basis $\eta_{1}, \ldots, \eta_{n}$ for $\overline{\operatorname{Lin}\left\{\pi_{1}(G) \xi_{1}\right\}}$. For a given $g \in G$, write

$$
\pi_{1}(g) \xi_{1}=\sum_{j=1}^{n} \alpha_{j}(g) \eta_{j}
$$

for some $\alpha_{1}(g), \ldots, \alpha_{n}(g)$ with $\sum\left|\alpha_{j}(g)\right|^{2}=\left|\xi_{1}\right|^{2} \leq 1$. Observe that

$$
\sum_{j=1}^{n} \alpha_{j}(g) \eta_{j}(x, y)=\left(\pi_{1}(g) \xi_{1}\right)(x, y)=\left(\pi(g) \xi_{1}\right)(x, y)=\xi_{1}\left(g^{-1} x, y\right)
$$

for every $(x, y) \in \tilde{Y}$. Since $\left|\xi_{1}\left(g^{-1} x, y\right)\right|=C$, for $(x, y) \in \tilde{Y}$ with $x=g(y)$, we conclude that $\sum_{j=1}^{n}\left|\eta_{j}(x, y)\right| \geq C>0$, for $(x, y)$ with $x=g y$ and, thus, for any $(x, y) \in \tilde{Y}$. This implies that the function $\sum_{j=1}^{n}\left|\eta_{j}(x, y)\right|$ is not integrable with respect to $\tilde{\mu}$. This contradiction yields that $\pi_{1}=0$ and, thus, the representation $\pi$ is regular.

We finish this section by giving examples of groups admitting no compressible actions. We observe that even though the following proposition yields a result similar to that of Theorem 2.11, the underlying assumptions are different and not mutually interchangeable.

Proposition 2.13. Let $G$ be a countable group with trivial center and such that every proper quotient is finite or abelian. Assume that the group $G$ admits a compressible action on a regular Hausdorff space. Then all finite type non-regular representations of $G$ are of type $\mathrm{I}$.

Proof. Consider a non-regular indecomposable character $\chi$ of $G$. Let $(\pi, H, \xi)$ be the GNS-construction associated to $\chi$. By Proposition 2.7 there exists $g \neq e$ such that $|\chi(g)|=1$. Choose $h \in G$ not commuting with $g$. Denote by $N$ the normal subgroup of $G$ generated by the element $g h g^{-1} h^{-1}$. Using the arguments from the proof of Lemma 2.8 we obtain that $\pi \mid N=$ Id. Thus, the representation $\pi$ of the group $G$ gives rise to the representation of $G / N$ with the same von Neumann algebra. Recall that factor representations of abelian groups are scalar.

If a group $G$ as in the proposition above has a measure-preserving action on a measure space $(X, \mu)$ with $0<\mu(\operatorname{Fix}(g))<1$ for some $g \neq e$, then, in view of Theorem 2.12, such a group cannot have compressible actions. Examples of such groups are full groups of even Bratteli diagrams, commutators of topological full groups of Cantor minimal systems [6], and just infinite branch groups [7]. 


\section{Applications}

In this section we show that the results established in the previous section are applicable to Higman-Thompson groups and to the full groups of irreducible shifts of finite type.

\subsection{Higman-Thompson groups}

Definition 3.1. Fix two positive integers $n$ and $r$. Consider an interval $I_{r}=[0, r]$. Define the group $F_{n, r}$ as the set of all orientation preserving piecewise linear homeomorphisms $h$ of $I_{r}$ such that all singularities of $h$ are in $\mathbb{Z}[1 / n]=\left\{\frac{p}{n^{k}}: p, k \in \mathbb{N}\right\}$ and the derivative of $h$ at any non-singular point is $n^{k}$ for some $k \in \mathbb{Z}$.

Observe that the commutator subgroup of $F_{n, r}$ is a simple group and the abelianization of $F_{n, r}$ is isomorphic to $\mathbb{Z}^{n}$ [2], Section 4. Consider the subgroup $F_{n, r}^{0}$ of $F_{n, r}$ consisting of all elements $f \in F_{n, r}$ with $\operatorname{supp}(f)$ being a subset of $(0, r)$. Note that, in general, $\operatorname{supp}(f), f \in F_{n, r}$ might contain the endpoints of the interval $I_{r}$. Observe that (the commutator subgroup) $F_{n, r}^{\prime}=\left(F_{n, r}^{0}\right)^{\prime}$ [2], Section 4 . The following lemma shows that the commutator subgroup $F_{n, r}^{\prime}$ satisfies the assumptions of Theorem 2.9.

Lemma 3.2. The basis $\mathfrak{U}=\left\{(a, b):[a, b] \subset(0, r), a, b \in \mathbb{Z}\left[\frac{1}{n}\right]\right\}$ of the topology satisfies conditions (i)-(iv) of Definition 2.5 for the action of the group $R=\left(F_{n, r}^{0}\right)^{\prime}$ on $(0, r)$. Thus, the action of $R$ is compressible.

Proof. (1) Condition (i) and condition (iv) of Definition 2.5 are clearly satisfied.

To check condition (ii), consider intervals $U_{1}=(a, b)$ and $U_{2}=(c, d)$ both in $\mathfrak{U}$. Replacing $U_{2}$ by a subinterval if necessary we may assume that $\frac{b-a}{d-c}=n^{k}$ for some $k \in \mathbb{Z}$. Since the function $\frac{a-x}{c-x}$ is continuous in $x$ for $x \neq c$, we can find $x \in \mathbb{Z}\left[\frac{1}{n}\right]$ such that $0<x<\min \{a, c\}$ and $\frac{a-x}{c-x}=n^{k}$, for some $k \in \mathbb{Z}$. Similarly, we can find $y \in \mathbb{Z}\left[\frac{1}{n}\right], \max \{b, d\}<y<r$ such that $\frac{y-b}{y-d}=n^{k}$, for some $k \in \mathbb{Z}$. Let $h:[0, r] \rightarrow[0, r]$ be the function such that

$$
h(0)=0, \quad h(x)=x, \quad h(a)=c, \quad h(b)=d, h(y)=y, \quad h(r)=r,
$$

and $h$ is linear on each of the line segments $[0, x],[x, a],[a, b],[b, y],[y, r]$. Note that $h \in F_{n, r}$ and $h\left(U_{1}\right)=U_{2}$.

Set $m=\min (a, c)$ and $M=\max (b, d)$. Using the same arguments as above, find an element $f \in F_{n, r}$ with $f([m, M]) \subset(y, r)$. Then $g=f^{-1} h^{-1} f h \in R$ and $g\left(U_{1}\right)=U_{2}$.

(2) To check condition (iii), we consider intervals $U_{i}=\left(a_{i}, b_{i}\right), i=1,2,3$ from $\mathfrak{U}$ such that $\bar{U}_{1} \cap \bar{U}_{2}=\varnothing$. Without loss of generality, assume that $a_{1}>b_{2}$. Choose $r^{\prime}<r$ such that $U_{3} \cap\left(r^{\prime}, r\right)=\varnothing$. Using the ideas from (1) above, one can construct an element $g \in R$ such that $\operatorname{supp}(g) \cap\left(a_{2}, b_{2}\right)=\varnothing$ and $g\left(U_{2}\right) \subset\left(r^{\prime}, r\right)$. We leave the details to the reader. 
Observe that all finite factor representations of abelian groups are scalar representations, i.e. $\pi(g)=c_{g}$ Id, with $c_{g} \in \mathbb{T}$, the unit circle. In particular, the indecomposable characters of abelian groups are homomorphisms into $\mathbb{T}$.

Corollary 3.3. (1) The group $F_{n, r}^{\prime}$ has no proper characters.

(2) If $\chi$ is an indecomposable character of $F_{n, r}$, then $\chi$ is either regular or $\chi(g)=$ $\rho([g])$, where $[g]$ is the image of $g$ in the abelianization of $F_{n, r}$ and $\rho: \mathbb{Z}^{n} \rightarrow \mathbb{T}$ is a group homomorphism.

Proof. Statement (1) immediately follows from Lemma 3.2 and Theorem 2.9.

To establish the second result, we only need to check condition (2) of Theorem 2.11. Fix $g \in F_{n, r} \backslash\{e\}$. Find an interval $(a, b)$ with $g(a, b) \cap(a, b)=\varnothing$. Find a sequence of distinct elements $\left\{h_{n}\right\}_{n \geq 1} \subset\left(F_{n, r}\right)^{\prime}$ supported by $(a, b)$. Then $\left(h_{n}^{-1} g^{-1} h_{n}\right)\left(h_{m}^{-1} g h_{m}\right) \in\left(F_{n, r}\right)^{\prime}$ for any $n \neq m$. This completes the proof.

Definition 3.4. Let $n$ and $r$ be positive integers. Define Higman's group $G_{n, r}$ as the group of all right continuous bijections of $[0, r)$ which are piecewise linear, with finitely many discontinuities and singularities, all in $\mathbb{Z}[1 / n]$, slopes in $\left\{n^{k}: k \in \mathbb{Z}\right\}$, and mapping $\mathbb{Z}[1 / n] \cap[0, r)$ to itself.

Note that $F_{n, r} \subset G_{n, r}$. In fact, $F_{n, r}$ consists exactly of all continuous elements $g \in G_{n, r}$. In [8] Higman showed that the commutator subgroup $G_{n, r}^{\prime}$ is simple and that the abelianization of $G_{n, r}$ is trivial for even $n$ and is $\mathbb{Z} / 2 \mathbb{Z}$ for odd $n$.

Lemma 3.5. The groups $R=F_{n, r}^{\prime}$ and $G=G_{n, r}$ satisfy the conditions of Theorem 2.11 .

Proof. Corollary 3.3 shows that the group $R$ has no proper characters. Consider an arbitrary element $g \in G, g \neq e$. Choose an open interval $I$ such that $I \cap g^{-1}(I)=\varnothing$ and $g$ is continuous on both $I$ and $g^{-1}(I)$. It follows that for any two elements $r_{1}, r_{2} \in R$ with $\operatorname{supp}\left(r_{1}\right) \subset I, \operatorname{supp}\left(r_{2}\right) \subset I$ the element

$$
h=r_{2} g^{-1} r_{2}^{-1} r_{1} g r_{1}^{-1} \neq e
$$

is a continuous bijection of $[0, r)$. Observe that $h$ acts as the identity near 0 and $r$. It follows that $h \in R$ and the elements $r_{1} g r_{1}^{-1}$ and $r_{2} g r_{2}^{-1}$ belong to the same coset of $G / R$. Since the group $R$ has infinitely many elements supported by the set $I$, we immediately establish condition (ii).

The following result is an immediate corollary of Theorem 2.11 applied twice to the pairs $R=F_{n, r}^{\prime}, G=\left(G_{n, r}\right)^{\prime}$ and $R=F_{n, r}^{\prime}, G=G_{n, r}$.

Corollary 3.6. (1) The group $G_{n, r}^{\prime}$ has no proper characters.

(2) If $\chi$ is an indecomposable character of $G_{n, r}$, then $\chi$ is either regular or $\chi(g)=$ $\rho([g])$, where $[g]$ is the image of $g$ in the abelianization of $G_{n, r}$ and $\rho: G_{n, r} / G_{n, r}^{\prime} \rightarrow$ $\mathbb{T}$ is a group homomorphism. 
3.2. Full groups of irreducible shifts of finite type. We refer the reader to Section 6 of [11] for a comprehensive study of full groups of étale groupoids including the groups discussed below.

Let $(V, E)$ be a finite directed graph. Suppose that the adjacency matrix of the graph is irreducible and is not a permutation matrix. For an edge $e \in E$, denote by $i(e)$ the initial vertex and by $t(e)$ its terminal vertex. Set

$$
X=\left\{\left\{e_{n}\right\}_{n \geq 1} \in E^{\mathbb{N}}: t\left(e_{k}\right)=i\left(e_{k+1}\right) \text { for every } k \in \mathbb{X}\right\} .
$$

Equipped with the product topology, $X$ is a Cantor set. We note that the space $X$ along with the left shift is called a one-sided subshift of finite type, see [11] and references therein regarding relations with symbolic dynamics.

An $n$-tuple $\left(e_{1}, \ldots, e_{n}\right) \in E^{n}$ is called admissible if $t\left(e_{k}\right)=i\left(e_{k+1}\right)$ for every $1 \leq k \leq n-1$. Two admissible tuples $\bar{e}=\left(e_{1}, \ldots, e_{n}\right)$ and $\bar{f}=\left(f_{1}, \ldots, f_{m}\right)$ are called compatible if $t\left(e_{n}\right)=t\left(f_{m}\right)$. Each admissible tuple $\bar{e}=\left(e_{1}, \ldots, e_{n}\right)$ defines a clopen set $U(\bar{e})=\left\{x \in X: x_{i}=e_{i}, i=1, \ldots, n\right\}$. Such clopen sets form a basis of the topology. Given two compatible admissible tuples $\bar{e}_{1}$ and $\bar{e}_{2}$, define a continuous map $\pi_{\bar{e}_{1}, \bar{e}_{2}}: U\left(\bar{e}_{1}\right) \rightarrow U\left(\bar{e}_{2}\right)$ as

$$
\pi_{\bar{e}_{1}, \bar{e}_{2}}\left(\bar{e}_{1}, x_{n+1}, x_{n+2}, \ldots\right)=\left(\bar{e}_{2}, x_{n+1}, x_{n+2}, \ldots\right) .
$$

Definition 3.7. Following [11], we define the full group of $X$, in symbols [[X]], as the set of all homeomorphisms $g$ of $X$ for which there exists two clopen partitions $X=\bigsqcup_{i=1}^{n} U\left(\bar{e}_{i}\right)=\bigsqcup_{i=1}^{n} U\left(\bar{f}_{i}\right)$ with $e_{i}$ and $f_{i}$ being compatible admissible tuples (possibly of different lengths), $i=1, \ldots, n$, such that $g \mid U_{\bar{e}_{i}}=\pi_{\bar{e}_{i}, \bar{f}_{i}}$ for every $i=1, \ldots, n$.

For a clopen subset $Y \subset X$, set $[[X \mid Y]]$ as the set of all $g \in[[X]]$ with $\operatorname{supp}(g) \subset Y$.

The following result was established in Lemma 6.1 and Theorem 4.16 of [11]

Proposition 3.8. For any clopen set $Y \subset X$, the commutator group $[[X \mid Y]]^{\prime}$ is simple.

Fix an arbitrary point $x_{0} \in X$. Find an increasing sequence of clopen sets $\left\{Y_{n}\right\}$ such that $X \backslash\left\{x_{0}\right\}=\bigcup_{n} Y_{n}$. Set $R=\bigcup_{n}\left[\left[X \mid Y_{n}\right]\right]^{\prime}$. It follows from Proposition 3.8 that the group $R$ is simple. Observe that the group $R$ consists of all elements $g \in[[X]]^{\prime}$ equal to the identity on some neighborhood of $x_{0}$.

Denote by $\mathcal{F}$ the set of all admissible tuples which are not prefixes of $x_{0}$. Define

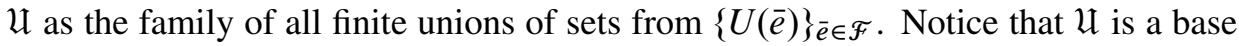
of the topology on $X \backslash\left\{x_{0}\right\}$. One can check that $\mathcal{U}$ satisfies conditions (i)-(iv) of Definition 2.5 for the action of $R$. Thus, using Theorem 2.9, we conclude that the group $R$ has no characters. Considering $R$ as a subgroup of $G=[[X]]$, one can check that the assumptions of Theorem 2.11 are satisfied. We leave the details to the reader.

Corollary 3.9. If $\chi$ is an indecomposable character of $[[X]]$, then $\chi$ is either regular or $\chi(g)=\rho([g])$, where $[g]$ is the image of $g$ in the abelianization of $[[X]]$ and $\rho:[[X]] /[[X]]^{\prime} \rightarrow \mathbb{T}$ is a group homomorphism. 
Applying Theorem 2.12, we also obtain the following result.

Corollary 3.10. Let $G$ be the commutator subgroup of either a Higman-Thompson group or the full group of an irreducible shift of finite type. Then every ergodic measure-preserving action of $G$ is essentially free.

To finish our discussion, we notice that the full group of the one-sided Bernoulli shift over the alphabet with $n$ letters is isomorphic to $G_{n, 1}$ [12].

Acknowledgements. We would like to thank R. Grigorchuk for the discussion of Higman-Thompson groups and for his valuable comments. We also thankful to the referee for his/her suggestions that improved the text.

\section{References}

[1] M. Abert, Y. Glasner, and B. Virag, Kesten's theorem for invariant random subgroups. Duke Math. J. 163 (2014), no. 3, 465-488. Zbl 06282535 MR 3165420

[2] K. Brown, Finiteness properties of groups. J. Pure Appl. Algebra 44 (1987), no. 1-3, 45-75. Zbl 0613.20033 MR 0885095

[3] J.W. Cannon, J. W. Floyd, and W. R. Parry, Introductory notes on Richard Thompson's groups. Enseign. Math. (2) 42 (1996), no. 3-4, 215-256. Zbl 0880.20027 MR 1426438

[4] A. Connes, Classification of injective factors. Cases $\mathrm{II}_{1}, \mathrm{II}_{\infty}, \mathrm{III}_{\lambda}, \lambda \neq 1$. Ann. of Math. (2) 104 (1976), no. 1, 73-115. Zbl 0343.46042 MR 0454659

[5] J. Feldman and C. Moore, Ergodic equivalence relations, cohomology, and von Neumann algebras. I. Trans. Amer. Math. Soc. 234, no. 2 (1977), 289-324. Zbl 0369.22009 MR 0578656

[6] A. Dudko and K. Medynets, On characters of inductive limits of symmetric groups. $J$. Funct. Anal. 264 (2013), no. 7, 1565-1598. Zbl 1271.20008 MR 3019724

[7] R. Grigorchuk, Some problems of the dynamics of group actions on rooted trees. Tr. Mat. Inst. Steklova 273 (2011), Sovremennye Problemy Matematiki, 72-191; English transl. Proc. Steklov Inst. Math. 273 (2011), no. 1, 64-175 Zbl 1268.20027 MR 2893544

[8] G. Higman, Finitely presented infinite simple groups. Notes on Pure Mathematics, No. 8 (1974), Department of Pure Mathematics, Department of Mathematics, I.A.S. Australian National University, Canberra 1974. MR 376874

[9] R. Dougherty, S. Jackson, and A. S. Kechris, The structure of hyperfinite Borel equivalence relations. Trans. Amer. Math. Soc. 341 (1994), 193-225. Zbl 0803.28009 MR 1149121

[10] A. Kirillov, Positive-definite functions on a group of matrices with elements from a discrete field. Dokl. Akad. Nauk SSSR 162 (1965), 503-505; English transl. Soviet Math. Dokl. 6 (1965), 707-709. Zbl 1271.20008 MR 0193183

[11] H. Matui, Topological full groups of one-sided shifts of finite type. Preprint, arXiv: 1210.5800 . 
[12] V. Nekrashevych, Cuntz-Pimsner algebras of group actions. J. Operator Theory 52 (2004), no. 2, 223-249. MR 2119267

[13] S. V. Ovchinnikov, Positive-definite functions on Chevalley groups. Funkcional. Anal. $i$ Priložen. 5 (1971), no. 1, 91-92. MR 0291371

[14] S. V. Ovchinnikov, Positive definite functions on Chevalley groups. Izv. Vysš. Ucebn. Zaved. Matematika (1971), no. 8 (111), 77-87 (in Russian). MR 0291372

[15] H. L. Skudlarek, Die unzerlegbaren Charaktere einiger diskreter Gruppen. Math. Ann. 233 (1976), 213-231. Zbl 0313.43010 MR 0463356

[16] M. Stein, Groups of piecewise linear homeomorphisms. Trans. Amer. Math. Soc. 332 (1992), no. 2, 477-514. Zbl 0798.20025 MR 1094555

[17] M. Takesaki, Theory of operator algebras I. Operator algebras and non-commutative geometry 5, Encyclopaedia Math. Sci. 124, Springer-Verlag, Berlin 2002. Zbl 1059.46032 MR 0548728

[18] D. Voiculescu, Sur les representations factorielles finies de $\mathbb{U}(1)$ et autres groupes semblables. C. R. Acad. Sci. Paris Sér. A 279 (1974), 945-946. Zbl 0291.22006 MR 360924

[19] D. Voiculescu, Representations factorielles de type $\mathrm{II}_{1}$ de U(1). J. Math. Pures Appl. (9) 55 (1976), no. 1, 1-20. Zbl 0352.22014 MR 442153

[20] A. Vershik, Nonfree actions of countable groups and their characters. Zapiski Nauchn. Semin. POMI 378 (2010), 5-16; English transl J. Math. Sci. 174 (2011), no. 1, 1-6. Zbl 1279.37004 MR 2749291

[21] A. Vershik, Totally nonfree actions and the infinite symmetric group. Moscow Math. J. 12 (2012), no. 1, 193-212. Zbl 06126169 MR 2952431

Received December 28, 2012; revised February 2, 2013

A. Dudko, Department of Mathematics, Stony Brook University, NY 11794-3651, U.S.A.

E-mail: artem.dudko@stonybrook.edu

K. Medynets, Department of Mathematics, 572C Holloway Road, U.S. Naval Academy, Annapolis, MD 21402-5002, U.S.A.

E-mail: medynets@usna.edu 